\title{
Individuals seeking gender reassignment: marked increase in demand for services
}

\author{
Josephine Fielding, ${ }^{1}$ Christopher Bass ${ }^{1}$
}

BJPsych Bulletin (2018) 42, 206-210, doi:10.1192/bjb.2018.30

${ }^{1}$ Oxford Health NHS Foundation Trust Correspondence to Josephine Fielding (j.fielding2@nhs.net)

First received 6 Jul 2017, final revision 3 Jan 2018, accepted 14 Mar 2018

(C) The Authors 2018. This is an Open Access article, distributed under the terms of the Creative Commons Attribution-NonCommercial-ShareAlike licence (http://creativecommons.org/ licenses/by-nc-sa/4.0/), which permits non-commercial re-use, distribution, and reproduction in any medium, provided the same Creative Commons licence is included and the original work is properly cited. The written permission of Cambridge University Press must be obtained for commercial re-use.
Aims and method To describe the pattern of referrals and characteristics of people aged over 18 seeking gender reassignment in Oxfordshire over a 6-year period (20112016).

Results A total of 153 individuals attended for assessment (of 162 referred); 97 (63.4\%) were natal males and 56 (36.6\%) were natal females. Mean age at referral was markedly different between the two groups, with females being younger. The number of referral significantly increased over the time period, by an average of $18 \%$ per year (95\% Cl 1.08-1.30). Eighty-seven patients sought transition from male to female, and 46 from female to male, while a smaller group $(n=13)$ had non-binary presentations. Twelve patients (7.8\%) had autism spectrum disorder.

Clinical implications There are various possible reasons for the increased demand for services for people with gender dysphoria, which we discuss here. When planning services in the UK, both the increase in overall referral rates and the apparent earlier age at referral should be taken into account.

Declaration of interest None.

Keywords Gender dysphoria; sexual and gender identity disorders; transsexualism.
Gender dysphoria is the personal experience of dissonance between assigned and desired gender. It has always been considered to be a rare disorder, but in recent years there has been an extraordinary increase in the number of referrals to both adult and child and adolescent gender clinics, with services becoming overwhelmed. ${ }^{1-3}$ Five years ago, our group published a service evaluation of referrals to the adult (age 18 onwards) Oxford Gender Clinic between 2004 and $2009 .{ }^{4}$ In this 5 -year period, there were 56 referrals. We found levels of psychiatric morbidity to be lower than described in other samples, and there were significantly more individuals seeking male-to-female transition (70\%) than female-to-male transition (30\%). The natal females (those assigned female at birth) were significantly younger than their natal male counterparts at referral. However, since 2011, there has been an increase in referrals to gender clinics, in particular to child and adolescent services, ${ }^{2,3}$ which has been noted in systematic reviews of prevalence as well as being the subject of considerable discussion in the media. ${ }^{5,6}$ We wanted to determine whether a similar pattern was occurring in services designed to cater for individuals aged 18 and over.

All individuals who request treatment for gender dysphoria in Oxfordshire are referred to a single clinician (C.B.) for psychiatric assessment and subsequent referral to a specialist centre. We aimed to review case notes for all referrals from 2011 until 2016 and to answer the following questions.
(1) Have referral patterns changed over time?

(2) If so, what are the main demographic and clinical characteristics?

We also discuss possible reasons for any changes in referral patterns.

\section{Method}

Individuals referred to C.B. between January 2011 and December 2016 were identified from a referral database kept by the Gender Clinic based in the Emergency Department Psychiatric Service at the John Radcliffe Hospital in Oxford. C.B. and J.F. reviewed case notes, and data were extracted and entered into a spreadsheet. All patients who had attended an appointment had had a full psychiatric assessment, as well as a review of any available medical records, such as the general practitioner (GP) summary. All patients were asked at assessment about current or past hormone therapy (prescribed or non-prescribed).

Patients were excluded if they were seen for follow-up only (i.e. they had been referred prior to the start of data collection) or if they had been referred after having previously transitioned. Two patients were also excluded owing to rare conditions: as this was a service evaluation, specific consent was not required, and the small sample size and unusual nature of the conditions meant that there would be concerns about the individuals being potentially identifiable. Data on age at referral were analysed with independent sample 
$t$-tests using IBM SPSS Statistics version 24.0, and the relationship between year of referral and numbers of referrals was examined using a negative binomial regression model in Stata, version 12 (StataCorp).

\section{Results}

\section{Demographic characteristics}

From a total of 164 referrals received, 153 individuals attended for assessment. The majority of patients were referred for initial assessment, and the 'did not attend' rate was low at $5.6 \%$.

The numbers of referrals, in 6-monthly intervals, between 2011 and 2016 are shown in Fig. 1.

Analysis of the numbers of referrals over time from 2011 to 2016, with data in 6-monthly intervals, was performed using a negative binomial regression model. This showed that the relationship between the numbers of referrals and year was significant at $P<0.001$. The incidence rate ratio was 1.18 (95\% CI 1.08-1.30), meaning that, on average, each year saw an average increase in the number of referrals of $18 \%$. When the analysis was repeated separately by natal sex, the number of referrals of both natal males and females increased significantly over time, but there was no significant difference between the natal sexes in the relationship between number of referrals and time.

The increase appeared most marked in the second half of the time period: the number of referrals nearly doubled over the 6 years, with 59 referrals from 2011 to 2013 and 103 from 2014 to 2016. Furthermore, mean ages were significantly lower in the latter 3 years compared with the first 3 years (a mean of 5.7 years younger, $t(112)=2.4, P=0.018$ ) (Fig. 2).

\section{Gender characteristics}

Of the 153 individuals who attended for assessment, markedly more were natal males $(97,63.4 \%)$ compared with the $56(36.6 \%)$ who were natal females (Fig. 3). The natal females were significantly younger than their male counterparts at referral: there was a mean difference of 10.5 years $(t(160)=5.5, P<0.001)$, with a mean age of 34.4 years for males and 24.3 years for females. This is consistent with the findings of our previous study; see Table 1 in Saunders and Bass (2011). There was no significant difference in the overall age of the sample compared with the previous study $(t(209)=0.98, P=0.33)$.

Eighty-seven individuals (56.9\%) were seeking to transition from male to female, and $46(30.1 \%)$ from female to male, while 13 (8.5\%) had non-binary gender identities. All but one of these 13 individuals were seen from 2014 to 2016. Overall, 146 patients $(95.4 \%$ of those attending the clinic) were assessed as having gender dysphoria.

The remainder $(n=7,4.6 \%)$ had a number of problems or reasons for referral that were not assessed as gender dysphoria (including body dysmorphic disorder, homosexual identity, discomfort with certain aspects of gender identity but not to the extent of gender dysphoria, fetishistic transvestism, and ideas about gender related to psychotic beliefs).

\section{Hormone therapy}

Twenty individuals (13.1\%) reported taking hormone replacement therapy at the time of assessment, with an additional five who had had hormones at some time in the past, plus two who had been prescribed finasteride for male pattern baldness. Of the 25, six were taking or had taken male hormones (such as testosterone), and 19 were taking or had taken female hormones (such as oestrogens).

\section{Educational level}

Notably, perhaps partly because of the geographical location of the study, a high proportion of our sample (39.2\%) had either been educated at BA degree level or higher (34, $22.2 \%$, including those currently undertaking postgraduate study) or were current undergraduate university students $(26,17.0 \%)$.

\section{Comorbidities}

Of those who attended for assessment, $60(39.2 \%)$ had a current psychiatric comorbidity, and $81(52.9 \%)$ had a past history of mental illness. Twelve $(7.8 \%)$ had a diagnosis of autism spectrum disorder (ASD). Depression was the most common mental disorder overall; 26 (17.0) had a current episode of depression and 59 (38.6) had had at least one previous episode. Seven patients (4.6\%) also reported a current or past history of self-harm. Other comorbidities are reported in Table 2.
Number of referrals

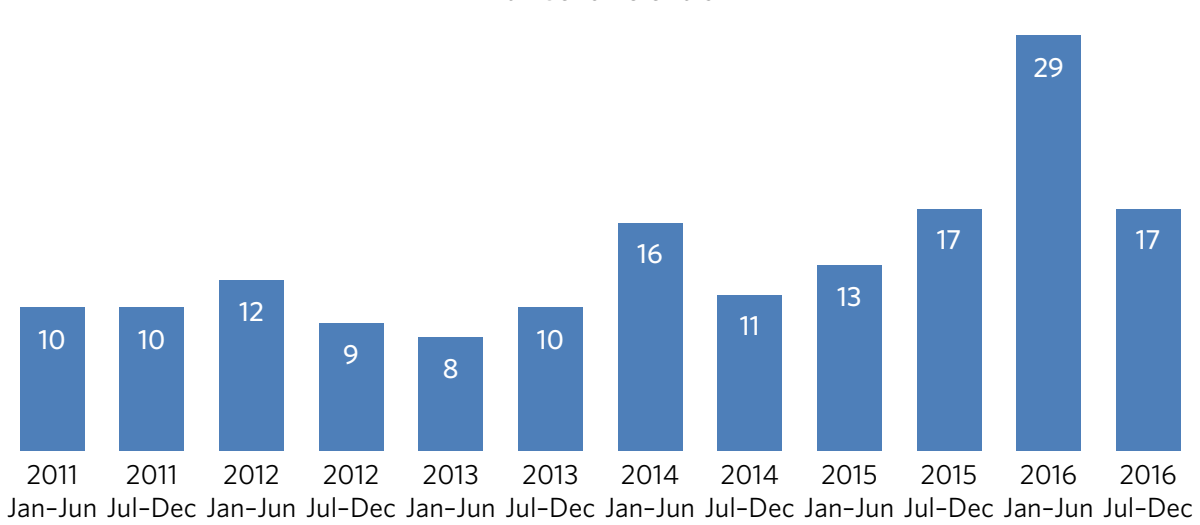

Referrals for gender dysphoria from 2011 to 2016

Jan-Jun Jul-Dec Jan-Jun Jul-Dec Jan-Jun Jul-Dec Jan-Jun Jul-Dec Jan-Jun Jul-Dec Jan-Jun Jul-Dec 
Mean age at referral

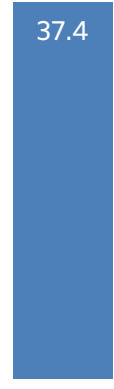

2011

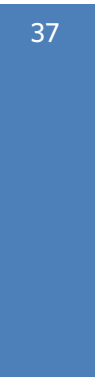

2012

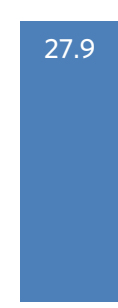

2013

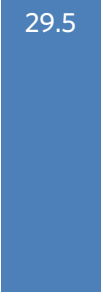

2014

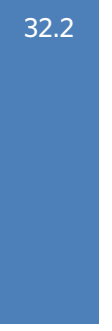

2015

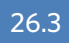

2016
Fig. 2 Mean age at referral.

\section{Outcomes}

The majority of patients ( $n=135,88.2 \%$ ) were referred on to a specialist referral centre for further assessment.

Of those not referred, 11 (7.2\%) had gender dysphoria but were not ready to pursue transition or onwards referral was considered inappropriate. For example, some did not wish to disclose their transgender identity to their spouses, or felt that transition would be unacceptable to their social networks given their cultural backgrounds. Of the remaining seven patients described above who were not thought to be transgender, six (3.9\%) were not referred but were offered alternative treatment or discharged, and one (0.65\%) was nevertheless referred on to a specialist gender clinic for a second opinion.

\section{Discussion}

\section{Summary of findings}

In a study of 162 patients referred to the Oxford Gender Clinic between 2011 and 2016, we report a significant increase in the number of referrals over the 6-year period, despite no changes in local policy or funding of the clinic. In particular, there is no designated endocrine clinic for patients to access in Oxford. The increase was marked in the past 3 years, with 103 referrals from 2014 to 2016 compared with 59 from 2011 to 2013. Similar findings have been reported in child and adolescent services. ${ }^{2}$ Our experience is also consistent with recent findings from a paediatric endocrinology service: ${ }^{3}$ of 38 patients seen over a 13 -year period, $74 \%$ of referrals to the clinic occurred during the past 3 years. In our detailed case note review of the 153 patients who attended for assessment, we also found a high rate of psychiatric comorbidity (in particular, depression and ASD), consistent with existing literature.

\section{Strengths and limitations}

The strengths of our study were that there is a single point of access in Oxfordshire for onward referral to specialist gender clinics, so our case note review was able to capture all patients referred within a certain time period in this geographical area. We were also able to compare the results with those of our previous study in 2004-2009.

Limitations of our study were that the clinic's assessment does not include a structured diagnostic interview, and although previous medical records were available in some cases, this was variable. Patients who had recently moved to the area, for example, might have had incomplete information in their local records, which might have particularly affected the large student population. Some information, such as on the number of patients taking hormones, was largely gathered through self-report, which may lead to underestimation.

\section{Consideration of reasons for changes in referral patterns}

Examination of our referrals in more detail revealed that the patterns of referrals changed during the 6-year period, with mean age at referral decreasing. The prevalence of non-

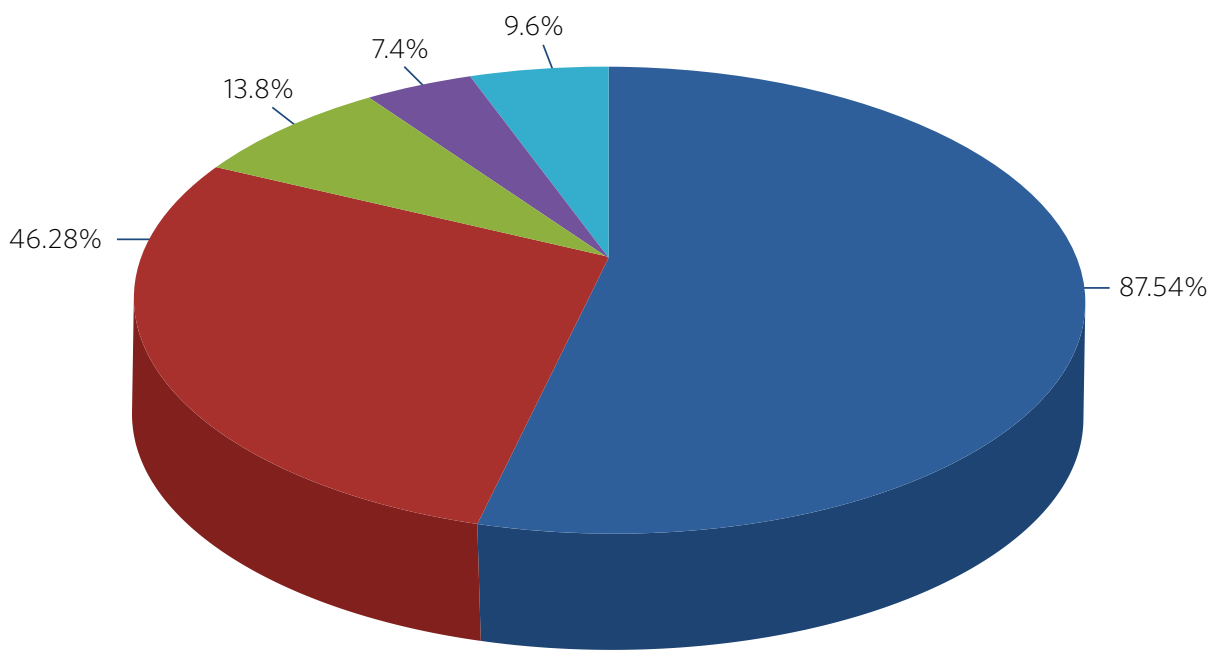

Male to female $\square$ Female to male Non-binary $\square$ Not transgender $\square$ Did not attend, so not diagnosed

Fig. 3 Diagnoses 
Table 1 Mean ages of all patients referred 2011-2013 and 2014-2016

\begin{tabular}{|ccccc} 
Referral period & $n$ & Mean age & s.d. & s.e.m. \\
\hline $2011-2013$ & 59 & 34.37 & 14.953 & 1.947 \\
\hline $2014-2016$ & 103 & 28.70 & 13.642 & 1.344 \\
\hline
\end{tabular}

binary gender presentations also increased during the 6-year period, echoing the phenomenon discussed by Richards et $a l^{7}$ This may reflect a change in how people's gender identities are developing, possibly because of the increased availability of information about non-binary genders from social media, the internet and peers. An alternative explanation might be that patients identified in this way previously, but perceived that if they disclosed this at assessment they might be denied treatment. It is conceivable that with greater awareness and less social stigma, these individuals may be developing more confidence about explaining their complex gender identities to professionals. The provision of comprehensive and accessible information to patients about options for gender identity and expression and possible medical interventions is crucial. ${ }^{8}$

We found similar rates of comorbid psychiatric disorders to those reported by others, ${ }^{9}$ with depression being the most common comorbidity. Like de Vries et $a l,{ }^{10}$ we found anecdotally that after patients had declared their gender dysphoria to others, or 'come out', their mood often improved and self-harming behaviour reduced in frequency

\begin{tabular}{|c|c|c|}
\hline Comorbidity & Current, n (\%) & Past, $n(\%)$ \\
\hline Any & $60(39.2)$ & $81(52.9)$ \\
\hline Depression & $26(17.0)$ & $59(38.6)$ \\
\hline Bipolar affective disorder & $3(2.0)$ & $0(0.0)$ \\
\hline Substance misuse - alcohol & $2(1.3)$ & $8(5.2)$ \\
\hline Substance misuse - drug & $1(0.65)$ & $8(5.2)$ \\
\hline Psychotic illness & $2(1.3)$ & $4(2.6)$ \\
\hline Post-traumatic stress disorder & $1(0.65)$ & $2(1.3)$ \\
\hline Generalised anxiety & $6(3.9)$ & $7(4.6)$ \\
\hline Social phobia & $9(5.9)$ & $4(2.6)$ \\
\hline Panic disorder & $3(2.0)$ & $3(2.0)$ \\
\hline Obsessive-compulsive disorder & $4(2.6)$ & $0(0.0)$ \\
\hline Other anxiety disorders & $1(0.65)$ & $1(0.65)$ \\
\hline Dissociative episodes & $0(0.0)$ & $1(0.65)$ \\
\hline Eating disorder & $0(0.0)$ & $4(2.6)$ \\
\hline Body dysmorphic disorder & $4(2.6)$ & $0(0.0)$ \\
\hline Autism spectrum disorder & $12(7.8)$ & $0(0.0)$ \\
\hline $\begin{array}{l}\text { Attention deficit hyperactivity } \\
\text { disorder }\end{array}$ & $5(3.3)$ & $1(0.65)$ \\
\hline Personality disorder & $2(1.3)$ & $4(2.6)$ \\
\hline Chronic somatoform disorder & $1(0.65)$ & $0(0.0)$ \\
\hline Learning disability & $2(1.3)$ & $0(0.0)$ \\
\hline
\end{tabular}

or ceased. Also, as in previously published literature, ${ }^{11}$ we found high rates of patients referred with a diagnosis of comorbid ASD (in our sample, about $8 \%$ of those attending for assessment). Although the assessment in the clinic does not include a structured diagnostic interview or a formal autism assessment, and hence our case note review only included previously established autism diagnoses, even this potential underestimate was still notably higher than the prevalence in the general population (estimated at 62 per 10000 in a worldwide review ${ }^{12}$ ). This echoes the findings of de Vries et al, ${ }^{10}$ who systematically assessed children and adolescents referred to a gender identity clinic and found an incidence of ASD of 7.8\%. They commented on the diversity of their findings, with both males and females having increased rates of ASD, and the variety of gender identity disorder diagnoses found in those individuals in their study with ASD. They speculated that in some individuals with ASD, feelings of gender dysphoria might be related to an experience of being 'different' generally and having difficulty with identity development, or to more rigid and concrete ideas about gender norms.

An unexpected finding was that only 20 (13.1\%) of patients were taking hormone treatment at the time of assessment, which is below the figure of $23 \%$ reported by Mepham et al. $^{13}$ Our study methods did not allow us to explain this discrepancy, although, like Mepham et al, proportionately more of our patients were transwomen taking oestrogens. Our findings do raise the possibility of underreporting, as hormone use was assessed through self-report, but this cannot be confirmed without more invasive methods such as blood tests, which were beyond the scope of our study. Another possibility would be that our sample truly had a lower rate of taking hormones, perhaps owing to an increased awareness of the medical risks of non-prescribed hormone treatment.

How can these recent marked increases in referral rates be explained? Our study does not provide any clues as to aetiology, but there are many possible explanations. An increase in referral rates can be due to an increase in the prevalence of the condition in the population, or to a change in help-seeking behaviour. Although our study does not provide evidence to differentiate between the two explanations, there have certainly been some recent cultural shifts which could influence the likelihood of patients with gender dysphoria seeking treatment. First, the public profile of transgender issues has been raised of late, with a recent increase in high-profile media figures such as Caitlyn Jenner and Chaz Bono coming out as transgender, and the broadcasting of a variety of documentaries about transgender people, including transgender children. ${ }^{14}$ The internet also provides much greater access to information about gender issues, so that individuals can research their condition and treatment options, and connect with others for support and advocacy. Many people, for example, document the story of their transition on YouTube to provide others with encouragement and advice from their personal experience. Lesbian, gay, bisexual and transgender (LGBT) support groups may also be more accessible because of the internet, and these also provide a source of peer support and information. Second, there have been campaigns to promote transgender people's rights and reduce discrimination 
in the UK and elsewhere, although significant stigma and discrimination do remain. ${ }^{15}$ All of this may have helped individuals with gender dysphoria to overcome barriers to seeking treatment, and potentially to presenting to healthcare professionals at an earlier age. Third, when patients present in primary care, or other contexts such as secondary mental healthcare, GPs and other health professionals have acquired a greater awareness of gender dysphoria and may be more likely to refer people for assessment.

However, decreased stigma and increasing access to information may not wholly account for changes in referral patterns, and additional factors may be needed to explain this. Advances in understanding the cause of gender dysphoria could help account for changes in prevalence; however, neurological studies investigating the possibility of structural or functional changes in the brains of those with gender dysphoria have as yet been inconsistent or inconclusive, ${ }^{16}$ and it is difficult to see how they could account for rapid changes of the nature seen in this and similar studies.

The implications of our findings support those of other recent studies, and together suggest that commissioners and others need to take into account the accelerating trend in referrals when planning services. People with gender dysphoria are distressed by long waiting lists and perceived health inequalities. ${ }^{17}$ In order to cope with increased demand, services need to provide multidisciplinary and interdisciplinary teams and networks that work and collaborate in the provision of services for people with gender dysphoria, as recommended in the Royal College of Psychiatrists' guidelines. ${ }^{18}$ Given the recommendations in ICD- $11^{19}$ that gender dysphoria be removed from the mental health section and renamed gender incongruence, a further question arises as to which medical discipline is most appropriate to provide these services.

\section{Acknowledgements}

We thank Achim Wolf for his invaluable help and advice on statistical analysis and comments on the manuscript, and Dr Kate Saunders for her work on the previous study.

\section{About the authors}

Josephine Fielding, MA, MRCPsych, is an Academic Clinical Fellow ST3 in Psychiatry at Oxford Health NHS Foundation Trust, John Radcliffe Hospital, Oxford, UK; Christopher Bass, MA, MD, FRCPsych, is at the Gender Clinic, Oxford Health NHS Foundation Trust, John Radcliffe Hospital, Oxford, UK.

\section{References}

1 Judge C, O'Donovan C, Callaghan G, Gaoatsme G, O'Shea D. Gender dysphoria - prevalence and comorbidities in an Irish adult population. Front Endocrinol 2014; 5: 87
2 Aitken $M$, Steensma $T$, Blanchard $R$, VanderLaan DP, Wood H, Fuentes $A$, et al. Evidence for an altered sex ratio in clinic-referred adolescents with gender dysphoria. J Sex Med 2015; 12: 756-63.

3 Chen M, Fuqua J, Eugster EA. Characteristics of referrals for gender dysphoria over a 13-year period. J Adolesc Health 2016; 58: 369.

4 Saunders K, Bass C. Gender reassignment: 5 years of referrals in Oxfordshire. BJPsych Bull 2011; 35: 325-7.

5 Arcelus J, Bouman WP, Van Den Noortgate W, Claes L, Witcomb G, Fernandez-Aranda F. Systematic review and meta-analysis of prevalence studies in transsexualism. Eur Psychiatry 2015; 30(6): 807-15.

6 Thompson A. We must catch up with the new sexual politics. The Times, 15 Feb 2015 (http://www.thetimes.co.uk/tto/opinion/columnists/article4571432.ece).

7 Richards C, Bouman WP, Seal L, Barker MJ, Nieder TO, T'Sjoen G Non-binary or genderqueer genders. Int Rev Psychiatry 2016; 28(1): 95-102.

8 Wylie K, Knudson G, Khan S, Bonierbale M, Watanyusakal S, Bara S. Serving transgender people: clinical care considerations and service delivery models in transgender health. Lancet 2016; 388: 401-11.

9 Dhejne C, Van Vierken R, Heylens G, Arcelus J. Mental health and gender dysphoria: a review of the literature. Int Rev Psychiatry 2016; 28: 4457.

10 de Vries A, Noens I, Cohen-Kettenis P, van Berckelaer-Onnes I, Doreleijers T. Autism spectrum disorders in gender dysphoric children and adolescents. J Autism Dev Disord 2010; 40: 930-6.

11 Glidden D, Bouman WP, Jones BA, Arcelus J. Gender dysphoria and autism spectrum disorder: a systematic review of the literature. Sex Med Rev 2016; 4(1): 3-14

12 Elsabbagh M, Divan G, Koh YJ, Kim YS, Kauchali S, Marcín C, et al. Global prevalence of autism and other pervasive developmental disorders. Autism Res 2012; 5: 160-79.

13 Mepham N, Bouman W, Arcelus J, Hayter M, Wylie K. People with gender dysphoria who self-prescribe cross sex hormones: prevalence, sources and side effects knowledge. J Sex Med 2014; 11: 1995-2001.

14. Transgender kids: who knows best? (http://www.bbc.co.uk/ programmes/b088kxbw).

15 Reisner S, Poteat T, Keatley J, Cabral M, Mothopeng T, Dunham E, et al. Global health burden and needs of transgender population: a review. Lancet 2016; 388: 412-36.

16 Smith ES, Junger J, Derntl B, Habel U. The transsexual brain - a review of findings on the neural basis of transsexualism. Neurosci Biobehav Rev 2015; 59: 251-66.

17 The National LGB\&T Partnership. Reducing health inequalities and improving access to health and social care for LGB\&T people. London, 2017.

18 Royal College of Psychiatrists. Good Practice Guidelines for the Assessment and Treatment of Adults with Gender Dysphoria (Council Report 181). RCPsych, 2013.

19 Reed G, Drescher J, Krueger R, Atalla E, Cochran SD, First MB, et al. Disorders related to sexuality and gender identity in the ICD-11: revising the ICD-10 classification based on current scientific evidence, best clinical practices, and human rights considerations. World Psychiatry 2016; 15: 205-21.

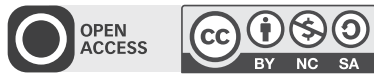

